\title{
Oncologist
}

\section{Clinical Use of Erythropoietic Stimulating Agents in Myelodysplastic Syndromes}

\author{
VALeria SANTini \\ Functional Unit of Haematology, AOU Careggi, University of Florence, Largo Brambilla 3, Firenze, Italy \\ Key Words. Myelodysplastic syndrome • Erythropoietin • IPSS low risk • ESAs • Refractory anemia \\ Disclosures: Valeria Santini: None. \\ The content of this article has been reviewed by independent peer reviewers to ensure that it is balanced, objective, and free from \\ commercial bias. No financial relationships relevant to the content of this article have been disclosed by the author or independent \\ peer reviewers.
}

\begin{abstract}
Myelodysplastic syndromes (MDS) are heterogeneous clonal diseases characterized by cytopenias resulting from ineffective hemopoiesis. Anemia affects the vast majority of patients with MDS and contributes substantially to their symptoms. For more than 20 years, recombinant human erythropoietin has been available for clinical use, and it has been employed in an attempt to relieve MDS-related anemia. Erythropoietin-alpha, erythropoietin-beta, and more recently darbepoetin have been found to increase hemoglobin levels and abolish transfusion dependence in $19 \%$ $68 \%$ of MDS cases. This wide range in clinical re-
\end{abstract}

sponse depends on several biological and clinical variables that allow the selection of patients with the highest probability of successful treatment. These agents are a mainstay in MDS therapy, but many issues are still open in terms of the initiation of therapy, the optimal dosage of erythropoietic stimulating agents (ESAs), the most efficient type of ESA, and the duration and outcome of such treatments. In this review, the mechanisms of response and predictive factors as well as an analysis of the clinical activity of ESAs in MDS therapy are presented. The Oncologist 2011;16(suppl 3):35-42

\section{INTRODUCTION}

Myelodysplastic syndromes (MDS) are a group of hematological neoplastic diseases with a heterogeneous clinical presentation and, most probably, heterogeneous pathophysiology. Their common shared clinical feature is the presence of severe peripheral blood cytopenias. In more than two thirds of MDS cases, anemia is present at diagnosis. Improving erythropoiesis, and thus eliminating fatigue and symptoms, is the main therapeutic target for the majority of MDS patients, because $>50 \%$ of patients present with ane- mia with an $\mathrm{Hb}$ level $<10 \mathrm{~g} / \mathrm{dL}$. Some $85 \%$ of patients develop more serious anemia as MDS progresses and $>80 \%$ require red blood cell (RBC) transfusion. In a mainly geriatric population of patients, anemia leads to co-morbidities that include cardiac complications, increased fatigue, diminished quality of life (QoL), and the need for chronic RBC transfusion. Well-known problems associated with RBC transfusions are iron overload, fluctuating hemoglobin levels, and persistently low hemoglobin levels (usually $<10 \mathrm{~g} / \mathrm{dL}$ ), in addition to the 


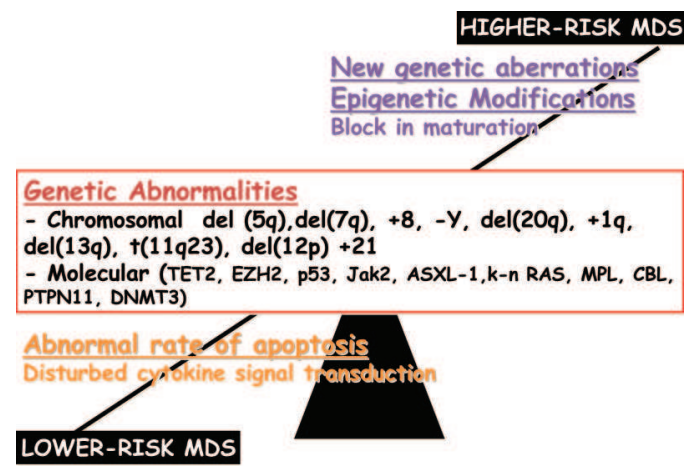

Figure 1. Myelodysplastic syndromes (MDS) patients are divided into a lower-risk group, in which apoptotic events in the marrow are prevalent and there is a defective response to cytokines (including erythropoietin), and a higher-risk group, in which a block in the maturation of marrow progenitors is the principal alteration.

intrinsic risks related to intolerance reactions, alloimmunization, and infections.

Diagnostic and prognostic evaluations of MDS patients are based on the French-American-British classification [1], the derived International Prognostic Score System (IPSS) [2], the World Health Organization (WHO) classification [3], or the derived WHO classification-based prognostic scoring system, WPSS [4]. These prognostic systems are strongly dependent on the morphological detection of immature cells and on the presence of cytogenetic abnormalities. The IPSS (which will be revised during this current year) divides MDS patients into a low-risk group (low and INT-1), in which apoptotic events in the marrow are prevalent and there is a defective response to cytokines (including erythropoietin [EPO]), and a high-risk group (intermediate-2 [INT-2] and high), in which a block in the maturation of marrow progenitors is the principal alteration (Figure 1). In the WPSS scoring system, transfusion dependence has been characterized as an important negative prognostic variable.

Despite all the above issues, the availability of these agents in clinics for more than 20 years, and the recommendations of several guidelines and consensus documents, ESAs are not approved by the Food and Drug Administration (FDA) or by the European Medicines Agency (EMA) for the treatment of anemia of MDS. The evidence for erythropoietic stimulating agent (ESA) activity and safety in MDS patients as well as pharmaco-economical arguments are examined in this review.

\section{Biological Studies}

Although the clinical use of ESAs in MDS patients is based on empiric observations of efficacy, some interesting studies have provided biological insights into the mechanisms of impaired response to endogenous EPO in MDS patients and the effects of pharmacological doses of ESAs.

It is a matter of debate whether the clinical response observed in $>50 \%$ of MDS cases is a result of proliferation and maturation of the dysplastic clone or stimulation of residual normal erythropoiesis by ESAs. Over the years, evidence has been provided to support both views. A few biological studies have been reported recently.

It has been known for more than two decades that bone marrow cells from MDS patients show an altered response to EPO in terms of in vitro colony formation. In particular, burst forming units erythroid (BFU-E) and colony-forming units erythroid (CFU-E) were found to be defective in cultures of unsorted and sorted CD34-positive bone marrow MDS cells, both after exposure to EPO and after exposure to EPO combined with interleukin-3 (IL-3), thrombopoietin, and other factors usually synergistic with EPO in terms of proliferative stimulus [5-7]. Only the addition of stem cell factor was found to be active to some extent [8]. Other functional parameters, such as EPO-dependent DNA synthesis and induction of GATA-1 binding activity, were also profoundly altered [9]. On the other hand, MDS progenitors showed lower expansion of multipotent progenitors (CFUGEMM) in semisolid culture systems, indicating a general disturbance of regulator cytokine production or response as the cause of such defects [10].

EPO-specific receptors have been demonstrated on MDS cells, and the percentage of bone marrow cells expressing the EPO receptor (EPO-R) from patients with MDS was comparable with that of normal marrow. No apparent correlation was evident between the number of MDS cells coexpressing the EPO-R and CD34 or CD71positive cells and impaired erythroid response, and MDS marrow cells expressed the full length EPO-R $[9,11]$. It was then postulated that intracellular structural defects of EPO-R, although not frequently analyzed and demonstrated [12], could cause defective receptor signaling following ligand binding [11]. In fact, STAT5-defective activation after EPO stimulation was observed in parallel with conserved STAT5 phosphorylation upon stimulation with IL-3, and this was attributed to a disturbance in an early stage of the EPO signal transduction pathway [9] (Figure 2).

On the other hand, the typical clinical characteristics of MDS-peripheral cytopenias with hyperproliferative marrow and ineffective hemopoiesis-appear to be related to the presence of excessive premature apoptosis of hematopoietic precursors. Several groups have demonstrated a higher number of apoptotic cells in the bone marrow of MDS patients [13-15]. Tumor necrosis factor alpha and interferon gamma are thought to participate in this apoptotic process [16]. Therefore, the typical dyserythropoiesis in 


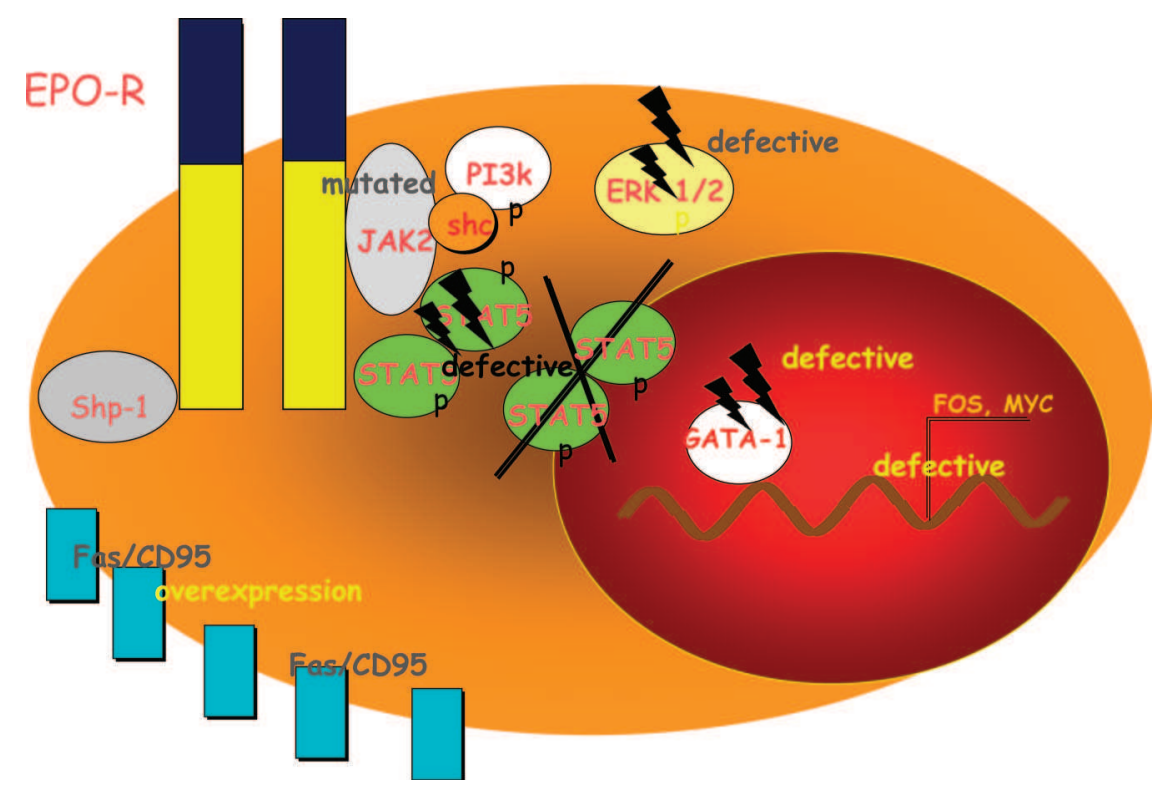

Figure 2. STAT5-defective activation after erythropoietin (EPO) stimulation was observed in parallel with conserved STAT5 phosphorylation upon stimulation with interleukin-3, and this was attributed to a disturbance in an early stage of the EPO signal transduction pathway.

MDS has been directly linked to greater expression of the transmembrane mediator of apoptotic cell death Fas/CD95 in the glycophorin A+ subpopulation [17] (Figure 2).

From this cumulative evidence, we may conclude that the ineffective erythropoiesis in MDS is caused by a defect in EPO-induced antiapoptotic pathways and by the stimulation of proapoptotic signals. Both obstacles to proliferation and maturation and to excessive apoptosis of erythroid precursors in MDS could be overcome by the use of pharmacological doses of EPO if we hypothesize that the dysplastic clones are the ones that are induced to differentiate and finally to recover.

The clinical observation that recently diagnosed MDS patients, who presumably have more residual polyclonal hematopoiesis, respond better to EPO treatment could argue against all the above-mentioned evidence of MDS progenitor sensitivity to ESAs. In this sense, the finding that EPO therapy reduces the number of cytogenetically abnormal clones in responding MDS patients, but not in unresponsive ones, is suggestive of a response to EPO attributable to karyotypically normal erythroid precursors, representing residual normal polyclonal hematopoiesis [18].

\section{Prediction of Response}

A rather large number of studies have addressed the possibility of identifying biological and clinical pretreatment criteria to predict response to EPO. The idea is to identify MDS patients who would most probably benefit from treat- ment, avoiding failures and/or inappropriately long and costly therapies.

\section{Biological Parameters}

Recently, flow cytometry analysis of MDS marrow has become more common. Although flow cytometry is still a method that requires specialist expertise and is not yet generally available in the routine diagnostic armamentarium, there are clear guidelines expressed by the European LeukemiaNet defining minimal combinations of antibodies to analyze aberrant immunophenotypes in individual cases of MDS. This enables the detection of altered numbers of CD34-positive precursors, aberrant expression of markers on myeloblasts, maturing myeloid cells, monocytes, or erythroid precursors, and the expression of lineage infidelity markers [19]. Such analyses of the pattern of expressed antigens revealed aberrant phenotypes of myeloblasts that were strongly associated with nonresponders among MDS patients with the greatest EPO response probability according to the predictive model of Hellström-Lindberg et al. (see below). This new predictive model may define three subgroups among IPSS low-risk MDS patients who will respond clinically to EPO treatment with a probability of $94 \%, 17 \%$, and $11 \%$, respectively [20].

Interestingly, in a small number of IPSS low-risk MDS patients (who constitute the ideal patient population for ESAs), the gene expression profile was investigated in sorted bone marrow glycophorin-positive erythroid cells and was found to correlate with their clinical response to 
EPO [21]. MDS case subjects who were nonresponsive to EPO showed repressed expression of genes responsible for proliferation/differentiation and DNA repair/stability, which were almost normally expressed in responders, in whom genes related to signal transduction, and in particular to the mitogen-activated protein kinase signaling pathway, seemed inhibited. This kind of analysis is now ongoing in a larger number of MDS cases, and it constitutes a challenging approach to the molecular mechanisms underlying ESA responsiveness.

In a much larger cohort of low/INT-1 MDS patients at diagnosis (127), MDS cases who were nonresponders to ESAs had a significantly lower number of BFU-E and CFU-E than responders. Moreover, EPO-dependent ERK1/2 activation was significantly lower in CD45neg/ CD71pos/GPAneg bone marrow cells from ESA nonresponders than ESA responders, and it could predict clinical response to ESAs [22].

Our group also found a correlation between EPO signal transduction alteration observed in vitro with a flow cytometry method and a lack of response to ESA treatment. In our study, CD71-positive marrow cells obtained from low/ INT-1 MDS patients not responding to ESAs demonstrated defective or absent EPO-driven STAT5 phosphorylation [23].

\section{Clinical Parameters}

EPO has been used in MDS since the late 1980s. During that time, several authors have focused on establishing response criteria to this agent, and analyses and meta-analyses of clinical trials with huge numbers of treated patients have led to some sound conclusions.

Very early observations indicated that MDS patients without a need for transfusion, with a serum EPO level $<200 \mathrm{U} / \mathrm{L}$, and a diagnosis other than refractory cytopenia with multilineage dysplasia with ringed sideroblasts (RARS) had a 68\% response rate to EPO. On the other hand, RARS patients with or without a need for transfusion but with a serum EPO level $>200 \mathrm{U} / \mathrm{L}$ had a response rate as low as 0\% [24]. Overall, the response to EPO ranged from a maximum of $68 \%$ to a minimum of $19 \%$ in unselected cases, excluding RARS cases. A meta-analysis of published studies (1990-2005) on ESA treatment [25] showed that low transfusion dependence, a morphological diagnosis of RA/RARS, the use of a fixed-dose versus weight-based EPO regimen, a shorter time from diagnosis to starting treatment, and a lower serum EPO level were the parameters predictive of response to ESA therapy.

An EPO level $>200 \mathrm{U} / \mathrm{L}$, transfusion dependence, an IPSS score of high and, in particular, $>10 \%$ blasts in the marrow, and a longer time elapsed from diagnosis were demonstrated to be negative predictive factors for response to EPO, EPO plus granulocyte colony-stimulating factor (G-CSF), and darbepoetin [26].

Although validated for therapy with EPO plus G-CSF [27], a practical scoring system was developed to select MDS patients with a high probability of response to treatment. Transfusion need and serum EPO level were still the variables considered.

The probability of response was in the range of $74 \%$ for a score of 0 to $23 \%$ for a score of 1 , and $7 \%$ for a score of 2 , where a score of 0 corresponds to a transfusion need $<2$ $\mathrm{U} / \mathrm{mo}$ and a serum EPO level $<500 \mathrm{U} / \mathrm{L}$ and higher levels for both transfusion need and EPO level are required for scores of 1 and 2 . In a recently reported study, a poorer response to ESAs was observed in MDS patients with the following characteristics: transfusion dependence, EPO level $>200 \mathrm{U} / \mathrm{L}$, a morphological diagnosis of refractory anemia with excess blasts (RAEB2) and refractory cytopenia with multilineage dysplasia with ringed sideroblasts (RCMDRS), and abnormal karyotype [28] (Table 1).

Although not much evidence is available and published in this regard, patients with a $5 \mathrm{q}$ chromosomal abnormality seem to respond to ESAs (both EPO and darbepoetin with or without G-CSF), with a significantly lower response rate and significantly shorter duration of response to both EPO and darbepoetin (with or without G-CSF) than MDS patients without del 5q [29]. Further evaluation of clinical response to ESA therapy in del $(5 q)$ patients is required. In this subgroup of patients, in cases of transfusion dependence, treatment with lenalidomide has been approved by the FDA, but not by the EMA. All parameters of response to ESAs for MDS patients are summarized in Table 1.

\section{Clinical Efficacy}

Soon after human recombinant EPO became available in clinics for renal failure patients, it was used for the treatment of anemia in MDS patients. At present, there are published reports of treatment with ESAs for $>2,500$ MDS patients. Quite recently, three meta-analyses addressed the efficacy of ESAs (EPO and darbepoetin) in MDS patients (3,025 and 2,530, respectively) [37]. Since 2000, International Working Group (IWG) criteria for the evaluation of response have been proposed in two subsequent versions (2000 and 2006), and since then, as unified and codified parameters of response are applied, evaluation and comparison of results from different studies are possible [31, 32].

A meta-analysis by Ross and colleagues pooled the data of 1,936 patients from 59 trials and included 5 studies (354 patients) randomizing Low-INT-1 IPSS risk MDS patients to either EPO with or without G-CSF or granulocyte mac- 
Table 1. Predictive variables for ESA response in MDS

Biological
Endogenous erythropoietin levels $<500 \mathrm{U} / \mathrm{L}$
Marrow blast $<10 \%$
IPSS low-INT-1
Diagnosis of refractory anemia
Normal karyotype
Clinical
Transfusion independence
Short duration of disease

Abbreviations: ESA, erythropoietic stimulating agents; INT-1, intermediate-1; IPSS, International Prognostic Score System; MDS, myelodysplastic syndromes.

rophage colony-stimulating factor (GM-CSF) or supportive care, wherein a relevant erythroid response was demonstrated in $27 \%$ of the patients. There were then reports of 51 EPO clinical studies and 3 darbepoetin single-arm studies. Response rates were higher $(32 \%-48 \%)$ in uncontrolled studies and even better after longer treatments [ $>20 \mathrm{wk}(\mathrm{s})]$ and with iron supplementation.

A second meta-analysis by Moyo et al. pooled data from 30 rigorously selected studies (of 79 published) treating MDS patients with rHuEpo-alpha (22 studies, 925 patients; 13 studies not employing IWG response criteria) or darbepoetin ( 8 studies, 389 patients), in monotherapy, but including recent clinical trials with higher ESA dosing. The pooled erythroid response rate was $43.9 \%$. For the studies that did not apply the IWG criteria, the response rate was as low as $31.6 \%$, whereas it was $57.6 \%$ for IWG-based studies. A comparison between erythropoietin darbepoetin was thus performed only for the IWG-based studies (9 EPOalpha and 8 darbepoetin), and no statistically significant difference was noted when the erythroid response rate was compared between the two agents at corresponding dosing regimens. On the other hand, higher dosing regimens of both EPO-alpha (60,000-80,000 U/wk) and darbepoetin (300 $\mu \mathrm{g} / \mathrm{wk}$ ) yielded greater erythroid responses. Regarding higher dosages of ESAs, several trials reported rates of erythroid response of $50 \%-71 \%$ in lower-risk MDS patients treated with high doses of rHuEpo $(60,000-80,000$ U/wk) [32, 33] $(3,334$ and 3,435) or darbepoetin $(300 \mathrm{mcg}$ once weekly or $500 \mu$ g every 2-3 weeks) $(3,536)$. Quite recently, it became evident that earlier inception of ESA therapy may delay the need for transfusion and substantially increase, for a longer period, the hemoglobin level $(63.1 \%$ response rate) [37]. On the other hand, it has been well known for some time that a longer duration of ESA treatment may recruit later responders, with a significant difference in the rate of response at 26 weeks versus 12 weeks [38].
At least a dozen studies have been published investigating the efficacy of the combination of EPO (or darbepoetin) plus G-CSF (or GM-CSF) in MDS patients, based on possible synergistic activity previously demonstrated, and claiming a higher rate of response to EPO as a single drug, mostly in transfusion-dependent and RARS patients. A meta-analysis performed by Mundle et al. specifically assessed the erythroid response to EPO-alpha monotherapy versus that obtained with EPO-alpha plus G-CSF or GMCSF. Fifteen studies fulfilled the criteria for the metaanalysis, enrolling 741 patients. The rates of erythroid response were comparable $(\sim 50 \%$ ) with the two treatment modalities. Higher doses of EPO-alpha induced a higher number of responses, superior to what was observed with standard dose EPO plus G-/GM-CSF [39].

The majority of clinical studies of ESAs in MDS patients use EPO-alpha. Few data have been published for EPO-beta, and these are mainly in combination with G-CSF or all-trans retinoic acid (ATRA). Recently, increasing numbers of investigators have tested the activity of darbepoetin, whose peculiar pharmacokinetics could produce different response rates $(3,840)$.

During ESA therapy, a conspicuous number of MDS patients may lose responsiveness. This may result from several factors, including depletion of iron, which occurs frequently. Normal iron levels should be restored by supplementation, even in cases of normal ferritin. Progression of MDS to IPSS higher risk/acute myeloid leukemia may demonstrate loss of sensitivity to ESAs but this could also indicate concomitant severe infections (production of cytokine suppressing erythropoiesis) or the development of autoimmune antierythrocyte antibodies.

No data are currently available regarding the efficacy of long-acting ESAs or EPO biosimilars in MDS patients.

Diverse therapeutic strategies and combinations of differentiation agents have been proposed to optimize the activity of ESAs in MDS patients. EPO has been combined with ATRA $[41,42]$, and good results were obtained for MDS patients who had failed previous ESA therapy (36\% response rate according to the IWG 2006 criteria) or who had transfusion dependence (response rate, 39\%). No advantage was seen for patients with an endogenous EPO level $>500$ U/L or with additional cytopenias. Other combinations, for example, EPO plus thalidomide and EPO plus lenalidomide, have been studied in a limited number of cases, the first with no advantage in anemia relief and the latter with a significantly higher incidence of thromboembolic events.

Some years ago, our group reported a sustained and rapid response to azacitidine combined with high-dose EPO-alpha in 10 IPSS INT-1 risk MDS patients [43]. Although safe as a combination therapy, it is not clear whether 
the improved erythropoiesis observed was a result of an effect of azacitidine or restoration of sensitivity to EPOalpha. No further systematic studies of such a combination have been published.

\section{SAFETY}

The use of ESAs in cancer, with or without chemotherapy, contributes to a lower need for transfusions and a higher hematocrit level, but these beneficial effects are not accompanied by longer survival, nor are they devoid of side effects, frequently caused by the presence of functional active receptors on neoplastic cells or on cells other than erythroid (i.e., endothelial) cells. In certain cases, ESAs have been demonstrated to be detrimental, because some reports suggested a shorter survival duration in patients with solid cancers treated with ESAs [44]. These observations, some years ago, prompted a critical reevaluation of ESA use in oncology practice, mainly in the U.S. Moreover, this evidence led the FDA to instruct the producers of ESAs to add a "black box" warning to the package insert of these agents. Consequently, the banning of their indiscriminate use in cancer in general, including MDS, was supported by a memo from the Centers for Medicare and Medicaid Services.

The evidence in MDS is, in fact, quite different. Regarding thromboembolic events during ESA treatment, there are few published reports involving MDS patients [45-47], whereas in several meta-analyses of clinical trials with EPO, including several thousands of patients, no episodes of hypertension, seizures, or cardiovascular events directly resulting from the growth factor were reported [25, 39, 48, 49]. In contrast, several studies indicated longer survival times in MDS patients responding to therapy with ESAs [26] (50 and 51).

Still, a proportion of patients, generally geriatric, with suspected MDS-related anemia are treated with ESAs on an empirical basis, without any proper diagnosis. This practice should be stopped, not only because of cost-effectiveness issues but also because we need more prospective, longterm studies of EPO versus placebo to definitively determine the safety of such therapy in MDS patients (52). Overall, the use of ESAs in MDS therapy has remained safe, provided published guidelines are followed.

\section{GUIDELINES}

The first systematic guidelines for the treatment of MDS patients were produced by the Italian Society of Hematology in 2002 (53). A recent update of these guidelines (54) devoted particular attention to recommendations regarding the correct use of ESAs, according to the evidence produced in the last several years. In particular, a grade B rec- ommendation indicates that patients with low-INT-1 IPSS risk MDS, with a hemoglobin level $<10 \mathrm{~g} / \mathrm{dL}$ and a serum erythropoietin level $<500 \mathrm{IU} / \mathrm{mL}$, should be considered for ESAs, that is, EPO-alpha, EPO-beta, or darbepoetin. The updated recommendations also consider follow-up of treatment, as reported below: "Fixed, rather than weight-adjusted, weekly subcutaneous doses of 60-80,000 U erythropoietin (once-a-week or subdivided in two doses) (grade A) or 300 mcg darbepoetin (once-a-week) should be used (grade B) for at least 12 weeks, possibly more than 20 (grade B). During ESA treatment, iron supplementation should be considered for patients with a transferrin saturation $<20 \%$ (grade D). If the patient responds to ESA treatment, an attempt should be made to reduce the dose (or the frequency of administration) to the lowest effective schedule able to maintain the hemoglobin level between 10 and $12 \mathrm{~g} / \mathrm{dL}$ (grade D). The combination of ESAs and G-CSF should be considered only for patients who are not heavily $(<2 U$ per month) red-cell transfusion dependent with serum erythropoietin levels $<500 \mathrm{mIU} / \mathrm{mL}$ and not responding to ESAs alone (grade C)" [54].

The last update of the 2011 National Comprehensive Cancer Network guidelines [55] acknowledges the fact that anemia is a major issue in MDS patients to be addressed even more carefully than in the past. An entire subsection of the guidelines is, in fact, dedicated to the evaluation of MDS-related anemia, therapy, and follow-up. Quite similarly to what was stated in the updated Italian Guidelines, in cases of symptomatic anemia, in IPSS low or INT-1 MDS patients, the driving parameter to choose treatment is indicated by the level of endogenous EPO. If EPO $<500 \mathrm{U} / \mathrm{L}$, the treatment of choice is EPO-alpha, 40,000-60,000 U, 1-3 times weekly subcutaneously (s.c.), or darbepoetin, $150-300 \mu \mathrm{g}$ weekly s.c. In cases of the presence of ringed sideroblasts or an absence of response, the addition of GCSF, $1-2 \mu \mathrm{g} / \mathrm{kg} 1-3$ times per week should be considered, as well as therapy with immunosuppressive or hypomethylating agents when endogenous EPO levels are >500 U/L.

Quite importantly, in 2007, the American Society of Hematology and the American Society of Clinical Oncology produced guidelines [56] for the correct use of ESAs in cancer patients. In this work, it was stressed that the only available evidence for the successful use of ESAs in anemia without chemotherapy was related to MDS. Only for MDS, in fact, had there been a randomized study [57] versus placebo (level of evidence II), although the lack of randomized trials evaluating EPO dosing was noted.

\section{Health Economy Assessment}

Although ESAs have been demonstrated to be effective at increasing hemoglobin levels and decreasing transfusion 
needs and cardiac comorbidities, with a subsequent improvement in QoL, the number of MDS patients with symptomatic anemia who require treatment is quite large and the economic burden of this type of therapy has to be carefully considered. The annual cost of therapy with EPO-alpha has been compared with that of darbepoetin and found to be less ( $\$ 26,076-\$ 52,176$ versus $\$ 41,904-\$ 87,300$ annually), with similar efficacy. Costs are equivalent whether firstline, second-line, or third-line treatment is analyzed and are in the same range of annual costs of azacitidine and decitabine, with lenalidomide treatment being more expensive [58]. Although the choice of treatment by physicians should not be biased by the cost of such treatment, the problem of sustainability arises in a disease like MDS, which mainly affects the geriatric population, which is steadily increasing in number in western societies. The optimization of treatment involves the good use of ESAs through strict selection of MDS patients with a high probability of response, based on the available and validated parameters. On the other hand, more studies are needed to clarify the economic burden of so-called "supportive care measures" [59] versus active therapy, balancing efficacy, outcome (survival), comorbidities, and, last but not least, the QoL of affected patients [60].

\section{REFERENCES}

1 Bennett JM. Classification of the myelodysplastic syndromes. Clin Haematol 1986;15:909-923.

2 Greenberg PL. Risk factors and their relationship to prognosis in myelodysplastic syndromes. Leuk Res 1998;22 Suppl 1:S3-S6.

3 Swerdlow S, Campo E, Harris N et al. WHO classification of tumors of hemopoietic and lymphoid tissues. Lyon: IARC Press, 2008.

4 Malcovati L, Germing U, Kuendgen A et al. Time-dependent prognostic scoring system for predicting survival and leukemic evolution in myelodysplastic syndromes. J Clin Oncol 2007;25:3503-3510.

5 Merchav S, Nielsen OJ, Rosenbaum $\mathrm{H}$ et al. In vitro studies of erythropoietin-dependent regulation of erythropoiesis in myelodysplastic syndromes. Leukemia 1990;4:771-774.

6 Asano H, Hotta T, Ichihara M et al. Growth analysis of marrow CD34positive hematopoietic progenitor cells in patients with myelodysplastic syndromes. Leukemia 1994;8:833-838.

7 Sawada K, Sato N, Notoya A et al. Proliferation and differentiation of myelodysplastic CD34+ cells: phenotypic subpopulations of marrow CD34+ cells. Blood 1995;85:194-202.

8 Backx B, Broeders L, Lowenberg B. Kit ligand improves in vitro erythropoiesis in myelodysplastic syndrome. Blood 1992;80:1213-1217.

9 Hoefsloot LH, van Amelsvoort MP, Broeders LC et al. Erythropoietininduced activation of STAT5 is impaired in the myelodysplastic syndrome. Blood 1997;89:1690-1700.

10 Merchav S, Nagler A, Fleischer-Kurtz G et al. Regulatory abnormalities in the marrow of patients with myelodysplastic syndromes. Br J Haematol $1989 ; 73: 158-164$.

11 Backx B, Broeders L, Hoefsloot LH et al. Erythropoiesis in myelodysplastic syndrome: expression of receptors for erythropoietin and kit ligand. Leukemia 1996;10:466-472.

12 Mittelman M, Gardyn J, Carmel M et al. Analysis of the erythropoietin receptor gene in patients with myeloproliferative and myelodysplastic syndromes. Leuk Res 1996;20:459-466.

13 Raza A, Mundle S, Iftikhar A et al. Simultaneous assessment of cell kinetics and programmed cell death in bone marrow biopsies of myelodysplastics reveals extensive apoptosis as the probable basis for ineffective hematopoiesis. Am J Hematol 1995;48:143-154.

14 Bouscary D, De Vos J, Guesnu M et al. Fas/Apo-1 (CD95) expression and apoptosis in patients with myelodysplastic syndromes. Leukemia 1997;11: $839-845$.

15 Parker JE, Fishlock KL, Mijovic A et al. 'Low-risk' myelodysplastic syn- drome is associated with excessive apoptosis and an increased ratio of proversus anti-apoptotic bcl-2-related proteins. Br J Haematol 1998;103: 1075-1082.

16 Kitagawa M, Saito I, Kuwata T et al. Overexpression of tumor necrosis factor (TNF)-alpha and interferon (IFN)-gamma by bone marrow cells from patients with myelodysplastic syndromes. Leukemia 1997;11: 2049-2054.

17 Fontenay-Roupie M, Bouscary D, Guesnu M et al. Ineffective erythropoiesis in myelodysplastic syndromes: correlation with Fas expression but not with lack of erythropoietin receptor signal transduction. Br J Haematol 1999; 106:464-473.

18 Rigolin GM, Porta MD, Bigoni R et al. rHuEpo administration in patients with low-risk myelodysplastic syndromes: evaluation of erythroid precursors' response by fluorescence in situ hybridization on May-GrunwaldGiemsa-stained bone marrow samples. Br J Haematol 2002;119:652-659.

19 van de Loosdrecht AA, Alhan C, Bene MC et al. Standardization of flow cytometry in myelodysplastic syndromes: report from the first European LeukemiaNet working conference on flow cytometry in myelodysplastic syndromes. Haematologica 2009;94:1124-1134.

20 Westers TM, Alhan C, Chamuleau ME et al. Aberrant immunophenotype of blasts in myelodysplastic syndromes is a clinically relevant biomarker in predicting response to growth factor treatment. Blood 2010;115:17791784.

21 Cortelezzi A, Colombo G, Pellegrini C et al. Bone marrow glycophorinpositive erythroid cells of myelodysplastic patients responding to highdose rHuEPO therapy have a different gene expression pattern from those of nonresponders. Am J Hematol 2008;83:531-539.

22 Frisan E, Pawlikowska P, Pierre-Eugene C et al. p-ERK1/2 is a predictive factor of response to erythropoiesis-stimulating agents in low/int-1 myelodysplastic syndromes. Haematologica 2010;95:1964-1968.

23 Spinelli E, Caporale R, Buchi F et al. Proliferative and apoptotic signalling in bone marrow cell subpopulations of MDS patients using flow cytometry technique. Haematologica 2010;95(suppl 2):219-220.

24 Hellström-Lindberg E. Efficacy of erythropoietin in the myelodysplastic syndromes: a meta-analysis of 205 patients from 17 studies. Br J Haematol 1995;89:67-71.

25 Moyo V, Lefebvre P, Duh MS, Yektashenas B, Mundle S. Erythropoiesisstimulating agents in the treatment of anemia in myelodysplastic syndromes: a meta-analysis. Ann Hematol 2008;87:527-536.

26 Park S, Grabar S, Kelaidi C et al. Predictive factors of response and survival in myelodysplastic syndrome treated with erythropoietin and G-CSF: the GFM experience. Blood 2008;111:574-582. 
27 Hellström-Lindberg E, Gulbrandsen N, Lindberg G et al. A validated decision model for treating the anaemia of myelodysplastic syndromes with erythropoietin + granulocyte colony-stimulating factor: significant effects on quality of life. Br J Haematol 2003;120:1037-1046.

28 Symeonidis A, Kouraklis-Symeonidis A, Zikos P, et al. Factors predicting for a favorable response among patients with myelodysplastic syndromes treated with erythropoietin \pm G-CSF. Leuk Res 2001;31:S116.

29 Kelaidi C, Park S, Brechignac S et al. Treatment of myelodysplastic syndromes with $5 q$ deletion before the lenalidomide era; the GFM experience with EPO and thalidomide. Leuk Res 2008;32:1049-1053.

30 Ross SD, Allen IE, Probst CA, Sercus B, Crean SM, Ranganathan G. Efficacy and safety of erythropoiesis-stimulating proteins in myelodysplastic syndrome: a systematic review and meta-analysis. Oncologist 2007;12: $1264-1273$.

31 Cheson BD, Bennett JM, Kantarjian $\mathrm{H}$ et al. Report of an international working group to standardize response criteria for myelodysplastic syndromes. Blood 2000;96:3671-3674.

32 Cheson BD, Greenberg PL, Bennett JM et al. Clinical application and proposal for modification of the International Working Group (IWG) response criteria in myelodysplasia. Blood 2006;108:419-425.

33 Stasi R, Brunetti M, Terzoli E et al. Once-weekly dosing of recombinant human erythropoietin alpha in patients with myelodysplastic syndromes unresponsive to conventional dosing. Ann Oncol 2004;15:1684-1690.

34 Spiriti MA, Latagliata R, Niscola P et al. Impact of a new dosing regimen of epoetin alfa on quality of life and anemia in patients with low-risk myelodysplastic syndrome. Ann Hematol 2005;84:167-176.

35 Latagliata R, Oliva EN, Volpicelli P et al. Twice-weekly high-dose rHuEpo for the treatment of anemia in patients with low-risk myelodysplastic syndromes. Acta Haematol 2008;120:104-107.

36 Gabrilove J, Paquette R, Lyons RM et al. Phase 2, single-arm trial to evaluate the effectiveness of darbepoetin alfa for correcting anaemia in patients with myelodysplastic syndromes. Br J Haematol 2008;142:379-393.

37 Park S, Kelaidi C, Sapena R et al. Early introduction of ESA in low risk MDS patients may delay the need for RBC transfusion: a retrospective analysis on 112 patients. Leuk Res 2010;34:1430-1436.

38 Terpos E, Mougiou A, Kouraklis A et al. Prolonged administration of erythropoietin increases erythroid response rate in myelodysplastic syndromes: a phase II trial in 281 patients. Br J Haematol 2002;118:174-180.

39 Mundle S, Lefebvre P, Vekeman F et al. An assessment of erythroid response to epoetin alpha as a single agent versus in combination with granulocyte- or granulocyte-macrophage-colony-stimulating factor in myelodysplastic syndromes using a meta-analysis approach. Cancer 2009; 115:706-715.

40 Kelaidi C, Fenaux P. Darbepoetin alfa in anemia of myelodysplastic syndromes: present and beyond. Expert Opin Biol Ther 2010;10:605-614.

41 Stasi R, Brunetti M, Terzoli E et al. Sustained response to recombinant human erythropoietin and intermittent all-trans retinoic acid in patients with myelodysplastic syndromes. Blood 2002;99:1578-1584.

42 Itzykson R, Ayari S, Vassilief D et al. Is there a role for all-trans retinoic acid in combination with recombinant erythropoetin in myelodysplastic syndromes? A report on 59 cases. Leukemia 2009;23:673-678.

43 Santini V, Gozzini A, Lunghi T et al. Azacitidine in combination with
$\mathrm{EPO}+\mathrm{G}-\mathrm{CSF}$ and valproic acid rapidly determines hematological improvement in pretreated non responsive IPSS INT-1 MDS patients. Leuk Res 2007;31:151.

44 Dicato M, Plawny L. Erythropoietin in cancer patients: pros and cons. Curr Opin Oncol 2010;22:307-311.

45 Rose EH, Abels RI, Nelson RA et al. The use of r-HuEpo in the treatment of anaemia related to myelodysplasia (MDS). Br J Haematol 1995;89:831837.

46 Steurer M, Sudmeier I, Stauder R et al. Thromboembolic events in patients with myelodysplastic syndrome receiving thalidomide in combination with darbepoietin-alpha. Br J Haematol 2003;121:101-103.

47 Niazy MN, Neyyarapally TI, Chattopadhyay A. Erythropoietin-induced deep vein thrombosis in myelodysplastic syndrome. J Assoc Physicians India 2008;56:195-196.

48 Jädersten M, Montgomery SM, Dybedal I et al. Long-term outcome of treatment of anemia in MDS with erythropoietin and G-CSF. Blood 2005; 106:803-811.

49 Casadevall N. Treatment of anaemia with rHuEpo in patients with MDS. Med Oncol 1998;15 Suppl 1:S35-S37.

50 Jädersten M, Malcovati L, Dybedal I et al. Erythropoietin and granulocytecolony stimulating factor treatment associated with improved survival in myelodysplastic syndrome. J Clin Oncol 2008;26:3607-3613.

51 Musto P, Villani O, Martorelli MC et al. Response to recombinant erythropoietin alpha, without the adjunct of granulocyte-colony stimulating factor, is associated with a longer survival in patients with transfusion-dependent myelodysplastic syndromes. Leuk Res 2010;34:981-985.

52 Steensma DP. Prospects and perils of retrospective risk assessment of erythropoiesis-stimulating agents. Am J Hematol 2008;83:693-694.

53 Alessandrino EP, Amadori S, Barosi G et al. Evidence- and consensusbased practice guidelines for the therapy of primary myelodysplastic syndromes. A statement from the Italian Society of Hematology. Haematologica 2002;87:1286-1306.

54 Santini V, Alessandrino PE, Angelucci E et al. Clinical management of myelodysplastic syndromes: update of SIE, SIES, GITMO practice guidelines. Leuk Res 2010;34:1576-1588.

55 Greenberg PL, Attar E, Bennet JM et al. Myelodysplastic syndromes. J Natl Compr Cancer Netw 2011;9:30-56.

56 Rizzo JD, Somerfield MR, Hagerty KL et al. Use of epoetin and darbepoetin in patients with cancer: 2007 American Society of Hematology/American Society of Clinical Oncology clinical practice guideline update. Blood 2008;111:25-41.

57 A randomized double-blind placebo-controlled study with subcutaneous recombinant human erythropoietin in patients with low-risk myelodysplastic syndromes. Italian Cooperative Study Group for rHuEpo in Myelodysplastic Syndromes. Br J Haematol 1998;103:1070-1074.

58 Greenberg PL, Cosler LE, Ferro SA et al. The costs of drugs used to treat myelodysplastic syndromes following National Comprehensive Cancer Network Guidelines. J Natl Compr Canc Netw 2008;6:942-953.

59 Santini V, Truschi T, Bertelli A. Cost of red blood cell transfusion: an activity-based cost analysis. Blood 2010;116:3817.

60 Caocci G, La Nasa G, Efficace F. Health-related quality of life and symptom assessment in patients with myelodysplastic syndromes. Expert Rev Hematol 2009;2:69-80. 